\title{
Revealing Explanation on Organic Dyes: A Review
}

\author{
Maitri Kagathara, Dipika J. Dalal, and Hitesh A. Solanki
}

\begin{abstract}
Dye is a substance which is used to colour various materials such as textiles, leather, and paper which is not altered with factors such as heat, light, washing, or any other exposure. However, the concept of dyes is completely different from the concept of pigments. Pigments in a nutshell can be defined as a material diffused in liquid forming a paint or ink. Dyes contain organic compound while pigments contain inorganic compound. Natural dyes have the privilege of causing minimal impact on environment, being renewable, sustainable, and safe. The paper here by aims to pile up the particulars of organic dyes, its history, types, sources, preparations and its medicinal use as well as pigments from which we get dyes. It further sheds some light on the comparison of natural dyes and synthetic dyes, concluding with unignorable advantages and disadvantages.
\end{abstract}

Index Terms- Environment, Medicinal value Organic dyes, Pigments.

\section{INTRODUCTION}

Green is probably the most common plant colour in most leaves. The green pigment chlorophyll in leaves helps to absorb the energy of the sun and turn it into chemical energy, which is then processed and used as plant food. Flowers colours are adaptations that attract insects and other species, which in effect pollinate and help replicate the plants. Some plants have colourful fruits which attract animals to eat them, spreading the seeds of the plant as they do so inadvertently. Scientists think other pigments may help protect plants from diseases. Given what we know about the role of a few thousand plant pigments, we still have a mystery about the role of most colours in plants (R.Siva, 2007).

While plants show a wide variety of colours, not all these pigments can be used as colours. Some do not dissolve in water, others cannot be adsorbed onto fibres, while others dissolve when washed or exposed to sunlight or air (R.Siva, 2007).

Designers used natural dyes as a design tool in a very effective way. The non-reproducibility and non-uniformity of shades make a unique piece of every development. Different

Manuscript revised on April 03, 2020 and published on April 10, 2020 Maitri Kagathara, Department of Botany, Bioinformatics and Climate Change Impacts Management, Gujarat University, Ahmedabad, India. Dipika J. Dalal, Research Scholar, Department of Botany, Bioinformatics and Climate Change Impacts Management, Gujarat University, Ahmedabad, India.

Dr. Hitesh A. Solanki, Department of Botany, Bioinformatics and Climate Change Impacts Management, Gujarat University, Ahmedabad, India. design techniques, such as tie-and-dye or sewing, resist, painting, stencilling, batik, Indian Ajrakh, Kalamkari, Ikat, etc., are being practiced by the designers in order to produce unique products (M. L. Gulrajani, 2001).

With over 9000 plants, Turkey is, from the flora standpoint, one of the richest countries in Europe and the Middle East. More than 3000 of these are known as endemics. The number of dye plants in Turkey is relatively high, as a contrast to this floristic richness (Ozlenen Erdem Ismal, 2016).

\section{History}

Natural colouring, colours, and dyeing are as common as textiles themselves. It was practised in Europe during the Bronze Age. Dating from 2600 BC, China has found the earliest written record of the use of natural dyes. Dyeing was established as early as in the Indus Valley period (2500 BC); this knowledge was confirmed by discoveries of coloured cloth garments and traces of madder dye in the ruins of Mohenjo-daro and Harappa's Indus Valley Civilization (3500 $\mathrm{BC})$.

Mummies covered in dyed cloth were discovered in Egypt. Chemical tests of red fabrics found in King Tutankhamen's grave in Egypt indicate alizarin, a pigment derived from madder, is present. In more modern times, when he defeated Susa, the Persian, Alexander the Great mentioned having found purple robes dated to $541 \mathrm{BC}$ in the royal treasury. Dyes such as woad, madder, weld, brasil wood, indigo and a deep reddish-purple were identified by the 4th century A.D. Brazil was named after where the woad was found (Gulrajani, M.L., 1992). Even before 2500 BC, Henna was used, while the Bible mentions saffron (M. L. Gulrajani, 2001).

In prehistoric times, for his cave paintings, man used to smash berries to mud pigment. During religious festivals as well as during battles, primitive people used plant dyes to paint animal skin and to their own bodies. They believed the colour would grant them magical powers, shield them from evil spirits and help them attain victory in battle (Oktav Bulut M \& Akar E, 2012).

Primitive dyeing methods included pressing plants into fabric to render or rub crushed pigments. With time and techniques, the methods were produced using natural dyes from crushed fruits, berries and other plants, which were boiled into the fabric and gave light and water fastness (resistance). Some of the well-known ancient dyes include madder, a red dye made from the Rubia tinctorum L. roots, blue indigo from the Indigofera tinctoria L. leaves, yellow from the saffron plant stigmas (Crocus sativus L.) and turmeric (Curcuma longa L.) (R.Siva, 2007). 
The Englishman Sir William Henry Perkin was only 17 when, in 1856, he found and produced the first synthetic organic dye (mauveine). The hue of Mauveine was identical to that of the ancient "Royal purple" (Tyrian purple) (Vanker, P.S., 2000).

\section{TYPES OF NATURAL DYES AND MORDANTS}

\section{A. Mordant}

Natural colours are substantive and require a mordant to adhere to the fabric, avoiding either fading with light exposure or washing out. Such compounds bind the natural tints to the textiles. Tints require mordants to help them adhere to fabric. If no mordants, such as lichens and walnut hulls, are required, they are called substantive dyes. These are called adjective dyes if they need a mordant. Common mordants are alum (usually used with tartar cream, which helps uniformity and brightens slightly); iron (or copper) (which saddens or dark colours, producing green shades); tin (usually used with tartar cream, which blooms or brightens our colours, especially reds, oranges and yellows) and blue vitriol (which saddens colours and brings out green shades) (R.Siva, 2007).

\section{B. Natural dyes are obtained from different plants}

With over 9000 plants, Turkey is, from the flora standpoint, one of the richest countries in Europe and the Middle East. More than 3000 of these are known as endemics. The number of dye plants in Turkey is relatively high, as a contrast to this floristic richness. In the regions where natural dyeing is alive, the colours obtained from the number of species have been described as follows: yellow from 84 species, green from 41 species, brown from 33 species, grey from 10 species, red from 7 species, pink from 5 species, violet from 4 species, blue from 3 species and black from 3 species (P Miller, 1759).

Most natural dyestuffs and stains were derived predominantly from plants and dominated as natural colour sources, creating various colours such as red, yellow, blue, black, brown and a mixture of these nearly all parts of plants such as root, bark, leaf, fruit, wood, seed, flower, etc. produce colouring. It is interesting to note that over 2000 pigments are synthesized through different parts of plants, only about 150 of which have been commercially exploited (R.Siva, 2007).

Table 1. Source of different coloured dyes and mordants (W Blith, 1652)

\begin{tabular}{|l|l|l|l|l|}
\hline $\begin{array}{l}\text { Botanical } \\
\text { name }\end{array}$ & $\begin{array}{l}\text { Common } \\
\text { name }\end{array}$ & $\begin{array}{l}\text { Colou } \\
\text { r }\end{array}$ & $\begin{array}{l}\text { Mordan } \\
\text { ts }\end{array}$ & $\begin{array}{l}\text { Parts } \\
\text { used }\end{array}$ \\
\hline $\begin{array}{l}\text { Mallotus } \\
\text { philippinensis } \\
\text { Muell. }\end{array}$ & Kamala & $\begin{array}{l}\text { Red } \\
\text { dye }\end{array}$ & Alum & Flower \\
\hline $\begin{array}{l}\text { Morinda } \\
\text { tinctoria } \text { L. }\end{array}$ & $\begin{array}{l}\text { Indian } \\
\text { mulberry }\end{array}$ & $\begin{array}{l}\text { Red } \\
\text { dye }\end{array}$ & Alum & Wood \\
\hline $\begin{array}{l}\text { Rubia } \\
\text { tinctorium } \text { L. }\end{array}$ & Madder & $\begin{array}{l}\text { Red } \\
\text { dye }\end{array}$ & Alum & Wood \\
\hline
\end{tabular}

\begin{tabular}{|c|c|c|c|c|}
\hline $\begin{array}{l}\text { Haematoxylon } \\
\text { campechianum } \\
\text { L. }\end{array}$ & Log wood & $\begin{array}{l}\text { Red } \\
\text { dye }\end{array}$ & - & Wood \\
\hline $\begin{array}{l}\text { Caesalpinia } \\
\text { sappan } \mathrm{L} \text {. }\end{array}$ & $\begin{array}{l}\text { Caesalpin } \\
\text { ia }\end{array}$ & $\begin{array}{l}\text { Red } \\
\text { dye }\end{array}$ & Alum & Wood \\
\hline $\begin{array}{l}\text { Carthamus } \\
\text { tinctorius L. }\end{array}$ & Safflower & $\begin{array}{l}\text { Red } \\
\text { dye }\end{array}$ & - & Flower \\
\hline $\begin{array}{l}\text { Rumex } \\
\text { dentatus } \mathrm{L} .\end{array}$ & $\begin{array}{l}\text { Khat } \\
\text { palak }\end{array}$ & $\begin{array}{l}\text { Red } \\
\text { dye }\end{array}$ & Alum & Wood \\
\hline $\begin{array}{l}\text { Solidago } \\
\text { grandis DC. }\end{array}$ & $\begin{array}{l}\text { Golden } \\
\text { rod }\end{array}$ & $\begin{array}{l}\text { Yello } \\
\text { w dye }\end{array}$ & Alum & Flower \\
\hline $\begin{array}{l}\text { Crocus sativus } \\
\mathrm{L} \text {. }\end{array}$ & Saffron & $\begin{array}{l}\text { Yello } \\
\text { w dye }\end{array}$ & Alum & Flower \\
\hline Tagetes sp. & Marigold & $\begin{array}{l}\text { Yello } \\
\text { w dye }\end{array}$ & Chrome & Flower \\
\hline $\begin{array}{l}\text { Butea } \\
\text { monosperma } \\
\text { (Lam) Taubert. }\end{array}$ & $\begin{array}{l}\text { Flame of } \\
\text { forest }\end{array}$ & $\begin{array}{l}\text { Yello } \\
\text { w dye }\end{array}$ & Alum & Flower \\
\hline $\begin{array}{l}\text { Alnus } \\
\text { glutinosa (L.) } \\
\text { Gaertn. }\end{array}$ & Alder & $\begin{array}{l}\text { Black } \\
\text { dye }\end{array}$ & $\begin{array}{l}\text { Ferrous } \\
\text { sulphate }\end{array}$ & Bark \\
\hline $\begin{array}{l}\text { Loranthus } \\
\text { pentapetalus } \\
\text { Roxb. } \\
\end{array}$ & $\begin{array}{l}\text { Rofblama } \\
\text { la }\end{array}$ & $\begin{array}{l}\text { Black } \\
\text { dye }\end{array}$ & $\begin{array}{l}\text { Ferrous } \\
\text { sulphate }\end{array}$ & Leaf \\
\hline $\begin{array}{l}\text { Terminalia } \\
\text { chebula } \text { Retz. }\end{array}$ & Harda & $\begin{array}{l}\text { Black } \\
\text { dye }\end{array}$ & $\begin{array}{l}\text { Ferrous } \\
\text { sulphate }\end{array}$ & Fruit \\
\hline $\begin{array}{l}\text { Anona } \\
\text { reticulata L. }\end{array}$ & $\begin{array}{l}\text { Custard } \\
\text { apple }\end{array}$ & $\begin{array}{l}\text { Black } \\
\text { dye }\end{array}$ & - & Fruit \\
\hline $\begin{array}{l}\text { Convallaria } \\
\text { majalis } \mathrm{L} .\end{array}$ & Lily & $\begin{array}{l}\text { Orang } \\
\text { e dye }\end{array}$ & $\begin{array}{l}\text { Ferrous } \\
\text { sulphate }\end{array}$ & Leaf \\
\hline Dhalia sp. & Dhalia & $\begin{array}{l}\text { Orang } \\
\text { e dye }\end{array}$ & Alum & Flower \\
\hline $\begin{array}{l}\text { Urtica dioica } \\
\text { L. }\end{array}$ & Nettles & $\begin{array}{l}\text { Orang } \\
\text { e dye }\end{array}$ & Alum & Leaf \\
\hline $\begin{array}{l}\text { Bixa orellena } \\
\text { L. }\end{array}$ & Annota & $\begin{array}{l}\text { Orang } \\
\text { e dye }\end{array}$ & Alum & Seed \\
\hline $\begin{array}{l}\text { Ligustrum } \\
\text { vulgare L. }\end{array}$ & Pivet & $\begin{array}{l}\text { Blue } \\
\text { dye }\end{array}$ & $\begin{array}{l}\text { Alum } \\
\text { and Iron }\end{array}$ & Fruit \\
\hline $\begin{array}{l}\text { Nymphaea } \\
\text { alba L. }\end{array}$ & Water lily & $\begin{array}{l}\text { Blue } \\
\text { dye }\end{array}$ & $\begin{array}{l}\text { Iron and } \\
\text { Acid }\end{array}$ & $\begin{array}{l}\text { Rhizom } \\
\mathrm{e}\end{array}$ \\
\hline $\begin{array}{l}\text { Isatis tinctoria } \\
\text { L. }\end{array}$ & Woad & $\begin{array}{l}\text { Blue } \\
\text { dye }\end{array}$ & - & Leaf \\
\hline
\end{tabular}

According to Table 1, below is the chart showing the percentage of parts used for dyes.

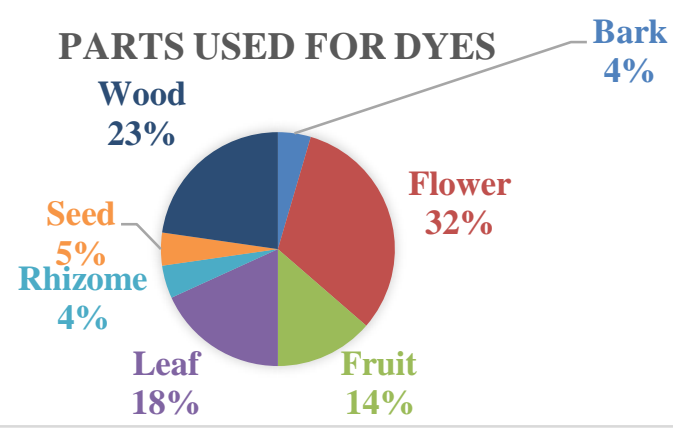

Here we have list of plants from which dye is extracted as well as they contain some medicinal value in table. 
International Journal of Research in Advent Technology, Vol.8, No.3, March 2020

E-ISSN: 2321-9637

Available online at www.ijrat.org

Table 2. Dye yielding plants (R.Siva, 2007)

\begin{tabular}{|c|c|c|c|c|c|c|c|c|c|}
\hline \multirow{2}{*}{$\begin{array}{l}\text { Botanical } \\
\text { Name }\end{array}$} & \multirow{2}{*}{ Family } & \multirow{2}{*}{$\begin{array}{l}\text { Commo } \\
\text { n name }\end{array}$} & \multirow{2}{*}{$\begin{array}{l}\text { Parts } \\
\text { Used }\end{array}$} & \multirow{2}{*}{ Colour } & \multirow{3}{*}{$\begin{array}{l}\text { Bauhinia } \\
\text { tomentosa } \\
\text { L. }\end{array}$} & \multirow[b]{3}{*}{$\begin{array}{l}\text { Caesalpinac } \\
\text { eae }\end{array}$} & & \multirow[b]{3}{*}{ Leaf } & \multirow[b]{3}{*}{ Yellow } \\
\hline & & & & & & & & & \\
\hline \multirow{2}{*}{$\begin{array}{l}\text { Abies } \\
\text { spectabilis } \\
\text { (D. Don.) } \\
\text { Spach. }\end{array}$} & \multirow{2}{*}{ Pinaceae } & \multirow{2}{*}{$\begin{array}{l}\text { East } \\
\text { Himalaya } \\
\mathrm{n} \quad \text { silver } \\
\text { fir }\end{array}$} & \multirow{2}{*}{ Cone } & \multirow{2}{*}{$\begin{array}{l}\text { Purple } \\
\text { or } \\
\text { Violet }\end{array}$} & & & & & \\
\hline & & & & & \multirow{2}{*}{$\begin{array}{l}\text { Bauhinia } \\
\text { variegate } \\
\text { L. }\end{array}$} & \multirow[b]{2}{*}{$\begin{array}{l}\text { Caesalpinac } \\
\text { eae }\end{array}$} & \multirow[b]{2}{*}{$\begin{array}{l}\text { Mahua } \\
\text { tree }\end{array}$} & \multirow[b]{2}{*}{ Bark } & \multirow[b]{2}{*}{ Yellow } \\
\hline $\begin{array}{l}\text { Acacia } \\
\text { catechu } \\
\text { (L.f.) Wild }\end{array}$ & $\begin{array}{l}\text { Mimosacea } \\
\text { e }\end{array}$ & $\begin{array}{l}\text { Cutch } \\
\text { tree }\end{array}$ & Bark & $\begin{array}{l}\text { Brown } \\
\text { / Black }\end{array}$ & & & & & \\
\hline $\begin{array}{l}\text { Acacia } \\
\text { dealbata } \\
\text { Link } \\
\end{array}$ & $\begin{array}{l}\text { Mimosacea } \\
\text { e }\end{array}$ & $\begin{array}{l}\text { Silver } \\
\text { wattle }\end{array}$ & Bark & $\begin{array}{l}\text { Brown } \\
\text { / Black }\end{array}$ & $\begin{array}{l}\text { Betula } \\
\text { utilis } \\
\text { D.Don }\end{array}$ & Betulaceae & $\begin{array}{l}\text { Himalaya } \\
\mathrm{n} \text { silver } \\
\text { birch }\end{array}$ & $\begin{array}{l}\text { Tree } \\
\text { gum }\end{array}$ & Brown \\
\hline $\begin{array}{l}\text { Acanthoph } \\
\text { onax } \\
\text { trifoliatum } \\
\text { (L.) Merr. }\end{array}$ & Araliaceae & & Fruit & Black & $\begin{array}{l}\text { D.Don } \\
\text { Briedelia } \\
\text { stipularis } \\
\text { L. }\end{array}$ & $\begin{array}{l}\text { Euphorbiac } \\
\text { eae }\end{array}$ & & Fruit & Black \\
\hline $\begin{array}{l}\text { (L.) Merr. } \\
\text { Actaea } \\
\text { spicata L. }\end{array}$ & $\begin{array}{l}\text { Ranunculac } \\
\text { eae }\end{array}$ & $\begin{array}{l}\text { Banberry } \\
\text { grape } \\
\text { wort }\end{array}$ & Seed & $\begin{array}{l}\text { Black, } \\
\text { red, } \\
\text { green }\end{array}$ & $\begin{array}{l}\text { Butea } \\
\text { monosper } \\
\text { ma (Lam) } \\
\text { Taubert. }\end{array}$ & $\begin{array}{l}\text { Papilonacea } \\
\mathrm{e}\end{array}$ & $\begin{array}{l}\text { Flame of } \\
\text { the forest }\end{array}$ & Flower & $\begin{array}{l}\text { Yello, } \\
\text { orange }\end{array}$ \\
\hline $\begin{array}{l}\text { Adathoda } \\
\text { vasica } \\
\text { Nees. }\end{array}$ & $\begin{array}{l}\text { Acanthacea } \\
\mathrm{e}\end{array}$ & Adalsa & Leaf & Yellow & $\begin{array}{l}\text { Caubert. } \\
\text { Caesalpini } \\
\text { a sappan }\end{array}$ & Caesalpinac & $\begin{array}{l}\text { Bastard } \\
\text { teak, } \\
\text { Benoal }\end{array}$ & Wood, & Red \\
\hline \multirow{2}{*}{$\begin{array}{l}\text { Aegle } \\
\text { marmelos } \\
\text { (L.) Corr. }\end{array}$} & \multirow[b]{2}{*}{ Rutaceae } & \multirow[b]{2}{*}{ Bael fruit } & \multirow{2}{*}{$\begin{array}{l}\text { Fruit } \\
\text { rind }\end{array}$} & \multirow[b]{2}{*}{ Yellow } & & & $\begin{array}{l}\text { Bengal } \\
\text { kino }\end{array}$ & & \\
\hline & & & & & $\begin{array}{l}\text { Carthamu } \\
s\end{array}$ & & & & \\
\hline \multirow{2}{*}{$\begin{array}{l}\text { Ailanthus } \\
\text { triphysa } \\
\text { (Dennst.) } \\
\text { Alston. }\end{array}$} & \multirow{2}{*}{$\begin{array}{l}\text { Simaroubac } \\
\text { eae }\end{array}$} & & \multirow[t]{2}{*}{ Leaf } & \multirow[t]{2}{*}{ Black } & $\begin{array}{l}S \\
\text { tinctorius } \\
\text { L. }\end{array}$ & Asteraceae & $\mathrm{r}$ & Flower & Yellow \\
\hline & & & & & $\begin{array}{l}\text { Cassia } \\
\text { auriculata }\end{array}$ & Caesalpinac & Tanner's & Flower, & Yellow \\
\hline \multirow{2}{*}{$\begin{array}{l}\text { Aloe } \\
\text { barbadens } \\
\text { is (L.) } \\
\text { Burm.f. }\end{array}$} & \multirow[b]{2}{*}{ Lilliaceae } & \multirow{2}{*}{$\begin{array}{l}\text { Curaco } \\
\text { aloe; } \\
\text { Indian } \\
\text { aloe }\end{array}$} & & & L. & & cassia & & \\
\hline & & & $\begin{array}{l}\text { Whole } \\
\text { plant }\end{array}$ & Red & $\begin{array}{l}\text { Cassia } \\
\text { occidental } \\
\text { is L. }\end{array}$ & $\begin{array}{l}\text { Caesalpinac } \\
\text { eae }\end{array}$ & $\begin{array}{l}\text { Negro } \\
\text { coffee }\end{array}$ & Seed & Brown \\
\hline $\begin{array}{l}\text { Althea } \\
\text { rosea Cav. }\end{array}$ & Malvaceae & $\begin{array}{l}\text { Holly } \\
\text { hock }\end{array}$ & Flower & Red & $\begin{array}{l}\text { Cassytha } \\
\text { filiformis }\end{array}$ & Lauraceae & & Stem & Brown \\
\hline Ardisia & & & & & & & & & \\
\hline $\begin{array}{l}\text { solanacea } \\
\text { Roxb. }\end{array}$ & $\begin{array}{l}\text { Myrstinace } \\
\text { ae }\end{array}$ & & Berry & Yellow & $\begin{array}{l}\text { Cedrela } \\
\text { toona }\end{array}$ & & & & \\
\hline $\begin{array}{l}\text { Arnebia } \\
\text { benthami } \\
\text { (Wall. ex } \\
\text { G. Don) }\end{array}$ & $\begin{array}{l}\text { Boraginace } \\
\text { ae }\end{array}$ & Pan & $\begin{array}{l}\text { Undergr } \\
\text { ound } \\
\text { parts }\end{array}$ & Purple & $\begin{array}{l}\text { Roxb. / } \\
\text { Toona } \\
\text { ciliata } \\
\text { Roem } \\
\end{array}$ & & $\begin{array}{l}\text { Red } \\
\text { Cedar }\end{array}$ & $\begin{array}{l}\text { Flower, } \\
\text { seed, leaf }\end{array}$ & $\begin{array}{l}\text { Yellow } \\
\text { /red }\end{array}$ \\
\hline $\begin{array}{l}\text { Arnebia } \\
\text { guttata }\end{array}$ & Boraginace & & Root & Red & $\begin{array}{l}\text { Citrus } \\
\text { medica } \mathrm{L} .\end{array}$ & Rutaceae & $\begin{array}{l}\text { Citron, } \\
\text { lime }\end{array}$ & Bark & Black \\
\hline Bunge & & & & & Clitoria & Fabaceae & & Flower & Blue \\
\hline Azadiracht & & & & & ternatea L. & & & & \\
\hline $\begin{array}{l}\text { a indica A. } \\
\text { Juzz }\end{array}$ & Meliaceae & Neem & Bark & Brown & $\begin{array}{l}\text { Cordia } \\
\text { myxa L. }\end{array}$ & $\begin{array}{l}\text { Boraginace } \\
\text { ae }\end{array}$ & & $\begin{array}{l}\text { Roots, } \\
\text { leaf }\end{array}$ & $\begin{array}{l}\text { Yellow } \\
\text {, red }\end{array}$ \\
\hline $\begin{array}{l}\text { Barleria } \\
\text { priontis L. }\end{array}$ & $\begin{array}{l}\text { Acanthacea } \\
\text { e }\end{array}$ & & Flower & Yellow & $\begin{array}{l}\text { Coscinium } \\
\text { fenestratu }\end{array}$ & & & Seed, & \\
\hline $\begin{array}{l}\text { Bassia } \\
\text { latifolia } \\
\text { Roxb. }\end{array}$ & Sapotaceae & Butter & Bark & Yellow & $\begin{array}{l}m \\
\text { (Gaertn.) } \\
\text { Clolebr. }\end{array}$ & $\begin{array}{l}\text { Mienisperm } \\
\text { aceae }\end{array}$ & $\begin{array}{l}\text { Iree } \\
\text { Turmeric }\end{array}$ & $\begin{array}{l}\text { bark, } \\
\text { wood }\end{array}$ & Red \\
\hline $\begin{array}{l}\text { IMadhuca } \\
\text { indica }\end{array}$ & Sapotale & tree & Dain & , brown & $\begin{array}{l}\text { Crocus } \\
\text { sativus L. }\end{array}$ & Iridaceae & Saffron & Flower & $\begin{array}{l}\text { Yellow } \\
\text { orange }\end{array}$ \\
\hline
\end{tabular}


International Journal of Research in Advent Technology, Vol.8, No.3, March 2020 E-ISSN: 2321-9637

Available online at www.ijrat.org

\begin{tabular}{|c|c|c|c|c|c|c|c|c|c|}
\hline $\begin{array}{l}\text { Cyanomet } \\
\text { ra } \\
\text { ramiflora } \\
\text { L. }\end{array}$ & $\begin{array}{l}\text { Caesalpinac } \\
\text { eae }\end{array}$ & & Wood & Black & $\begin{array}{l}\text { Indigofera } \\
\text { tinctoria } \\
\text { L. }\end{array}$ & Fabaceae & $\begin{array}{l}\text { Indian } \\
\text { indigo, } \\
\text { common } \\
\text { indigo }\end{array}$ & Leaf & $\begin{array}{l}\text { Blue, } \\
\text { blue-bl } \\
\text { ack }\end{array}$ \\
\hline $\begin{array}{l}\text { Dioscorea } \\
\text { bulbifera } \\
\text { L. }\end{array}$ & $\begin{array}{l}\text { Dioscoreac } \\
\text { eae }\end{array}$ & $\begin{array}{l}\text { Potato } \\
\text { yam, air } \\
\text { potato }\end{array}$ & Tuber & $\begin{array}{l}\text { Pale } \\
\text { colour }\end{array}$ & \multirow{2}{*}{$\begin{array}{l}\text { Jatropha } \\
\text { curcas L. }\end{array}$} & \multirow{2}{*}{$\begin{array}{l}\text { Euphorbiac } \\
\text { eae }\end{array}$} & \multirow{2}{*}{$\begin{array}{l}\text { Physic } \\
\text { nut, } \\
\text { purging } \\
\text { nut }\end{array}$} & \multirow{2}{*}{$\begin{array}{l}\text { Bark, } \\
\text { leaf }\end{array}$} & \multirow[t]{2}{*}{ Blue } \\
\hline \multirow{2}{*}{$\begin{array}{l}\text { Diospyros } \\
\text { embryopte } \\
\text { ris Pers. }\end{array}$} & \multirow{2}{*}{ Ebenaceae } & \multirow{2}{*}{$\begin{array}{l}\text { Gaub } \\
\text { persimm } \\
\text { on }\end{array}$} & \multirow[b]{2}{*}{ Fruit } & \multirow[b]{2}{*}{ Brown } & & & & & \\
\hline & & & & & \multirow{2}{*}{$\begin{array}{l}\text { Kirganelia } \\
\text { reticulate } \\
\text { (Poir) } \\
\text { Baill. } \\
\end{array}$} & \multirow[b]{2}{*}{$\begin{array}{l}\text { Euphorbiac } \\
\text { eae }\end{array}$} & & \multirow[b]{2}{*}{$\begin{array}{l}\text { Bark, } \\
\text { root }\end{array}$} & \multirow[b]{2}{*}{ Red } \\
\hline \multirow{2}{*}{$\begin{array}{l}\text { Dipteroca } \\
\text { rpus } \\
\text { turbinatus } \\
\text { Gaertn. }\end{array}$} & \multirow{2}{*}{$\begin{array}{l}\text { Dipterocarp } \\
\text { aceae }\end{array}$} & \multirow{2}{*}{$\begin{array}{l}\text { Common } \\
\text { Gurjan } \\
\text { tree }\end{array}$} & \multirow{2}{*}{$\begin{array}{l}\text { Twig, } \\
\text { bark }\end{array}$} & \multirow{2}{*}{$\begin{array}{c}\text { Yellow } \\
\text {, brown }\end{array}$} & & & & & \\
\hline & & & & & $\begin{array}{l}\text { Lawsonia } \\
\text { inermis L. }\end{array}$ & Lythraceae & Henna & Leaf & $\begin{array}{l}\text { Orange } \\
\text {, red }\end{array}$ \\
\hline \multirow[t]{2}{*}{$\begin{array}{l}\text { Elaeodend } \\
\text { ron } \\
\text { glaucum } \\
\text { (Rottb.) } \\
\text { Pers. } \\
\end{array}$} & \multirow[t]{2}{*}{$\begin{array}{l}\text { Celasterace } \\
\text { ae }\end{array}$} & & \multirow[t]{2}{*}{ Bark } & \multirow[t]{2}{*}{ Red } & $\begin{array}{l}\text { inermis L. } \\
\text { Lycopus } \\
\text { europaeus } \\
\text { L. }\end{array}$ & & $\begin{array}{l}\text { Gipsy } \\
\text { wort }\end{array}$ & Fruit & Green \\
\hline & & & & & Mallotus & & & & \\
\hline \multirow{2}{*}{$\begin{array}{l}\text { Eugenia } \\
\text { jambolana } \\
\text { Lam. }\end{array}$} & \multirow[t]{2}{*}{ Myrtaceae } & & \multirow[t]{2}{*}{$\begin{array}{l}\text { Bark, } \\
\text { leaf }\end{array}$} & \multirow[t]{2}{*}{ Red } & $\begin{array}{l}\text { philippien } \\
\text { sis Muell. }\end{array}$ & $\begin{array}{l}\text { Euphorbiac } \\
\text { eae }\end{array}$ & $\begin{array}{l}\text { Kamala } \\
\text { tree }\end{array}$ & Fruit & Red \\
\hline & & & & & Malphigia & Malpigiace & Barbedos & & \\
\hline $\begin{array}{l}\text { Euphorbia } \\
\text { tirucalli L. }\end{array}$ & $\begin{array}{l}\text { Euphorbiac } \\
\text { eae }\end{array}$ & & Wood & Red & glabra L. & ae & cherry & Hower & Yellow \\
\hline $\begin{array}{l}\text { Flemingia } \\
\text { congesta } \\
\text { Roxb. }\end{array}$ & Fabaceae & & Pod & $\begin{array}{l}\text { Red, } \\
\text { Yellow }\end{array}$ & $\begin{array}{l}\text { Melastom } \\
\text { a } \\
\text { malabathr } \\
\text { icum L. }\end{array}$ & & $\begin{array}{l}\text { Indian } \\
\text { rhododen } \\
\text { dron }\end{array}$ & Fruit & $\begin{array}{l}\text { Black, } \\
\text { purple }\end{array}$ \\
\hline $\begin{array}{l}\text { Galium } \\
\text { aparine L. }\end{array}$ & Rubiaceae & $\begin{array}{l}\text { Goose } \\
\text { grass }\end{array}$ & Root & Purple & $\begin{array}{l}\text { Michelia } \\
\text { champaka }\end{array}$ & Magnoliace & Champak & Flower & Yellow \\
\hline Galium & & & & Yellow & L. & & & & \\
\hline $\begin{array}{l}\text { rotundifoli } \\
\text { um L. }\end{array}$ & Rubiaceae & & Root & , brown & $\begin{array}{l}\text { Mimusops } \\
\text { elengi L. }\end{array}$ & Sapotaceae & $\begin{array}{l}\text { Bullet } \\
\text { wood }\end{array}$ & Bark & Brown \\
\hline $\begin{array}{l}\text { Galium } \\
\text { verum } \mathrm{L} .\end{array}$ & Rubiaceae & $\begin{array}{l}\text { Cheese } \\
\text { rennet }\end{array}$ & Root & $\begin{array}{l}\text { Yellow } \\
\text {, red }\end{array}$ & $\begin{array}{l}\text { Morinda } \\
\text { Citrifolia }\end{array}$ & Rubiaceae & & Root & Red, \\
\hline Garcinia & & & & & L. & Nuviacta & & Nove & Yellow \\
\hline $\begin{array}{l}\text { mangostan } \\
a \mathrm{~L} .\end{array}$ & Guttiferae & een & Fruit & Black & $\begin{array}{l}\text { Morinda } \\
\text { umbellata }\end{array}$ & Rubiaceae & & Root & Red \\
\hline Gardenia & & & & & & & & & \\
\hline $\begin{array}{l}\text { jasminoide } \\
s \mathrm{~J} \text {. Ellis. }\end{array}$ & Rubiaceae & $\begin{array}{l}\text { Cape } \\
\text { Jasmine }\end{array}$ & Fruit & Yellow & $\begin{array}{l}\text { Naregami } \\
a \quad \text { alata }\end{array}$ & & & Le: & Red \\
\hline $\begin{array}{l}\text { Garanium } \\
\text { wallichian }\end{array}$ & Geraniacea & Wallich & Fruit, & $\begin{array}{r}\text { Yellow } \\
\text { red, }\end{array}$ & $\begin{array}{l}\text { Wight \& } \\
\text { Arn. }\end{array}$ & Niel & & Leal & Ked \\
\hline um D.Don & & cranesbill & root & brown & Nyctanthe & & & & \\
\hline $\begin{array}{l}\text { Haematox } \\
\text { ylon } \\
\text { campechia }\end{array}$ & $\begin{array}{l}\text { Mimosacea } \\
\text { e }\end{array}$ & $\begin{array}{l}\text { Log } \\
\text { wood }\end{array}$ & $\begin{array}{l}\text { Heart } \\
\text { wood }\end{array}$ & Red & $\begin{array}{l}s \\
\text { arbortristi } \\
s \mathrm{~L} .\end{array}$ & Oleaceae & $\begin{array}{l}\text { Coral } \\
\text { jasmine }\end{array}$ & Flower & Yellow \\
\hline $\begin{array}{l}\text { campechia } \\
\text { num L. }\end{array}$ & & & & & Oldenland & & & & \\
\hline $\begin{array}{l}\text { Heliotropi } \\
\text { um } \\
\text { trigosum }\end{array}$ & Boraginace & & Leaf & Black & $\begin{array}{l}\text { la } \\
\text { umbellata } \\
\text { L. }\end{array}$ & Rubiaceae & $\begin{array}{l}\text { Chay-roo } \\
\text { t }\end{array}$ & Root & Red \\
\hline $\begin{array}{l}\text { trigosum } \\
\text { L. }\end{array}$ & & & & & Oxalis & Oxalidacea & Indian & Leaf & Blue \\
\hline $\begin{array}{l}\text { Indigofera } \\
\text { aspalathoi }\end{array}$ & Fabaceae & Wiry & Leaf & Blue-bl & $a \mathrm{~L}$. & & sorrel & Leal & Biue \\
\hline des Vahl. & ravacede & indigo & Leal & ack & $\begin{array}{l}\text { Papaver } \\
\text { rhoeas } \mathrm{L} .\end{array}$ & $\begin{array}{l}\text { Papaverace } \\
\text { ae }\end{array}$ & $\begin{array}{l}\text { Corn } \\
\text { poppy }\end{array}$ & Petal & Red \\
\hline $\begin{array}{l}\text { Indigofera } \\
\text { hirsuta } \mathrm{L} .\end{array}$ & Fabaceae & & Leaf & Indigo & $\begin{array}{l}\text { rhoeas L. } \\
\text { Peltophor }\end{array}$ & & & & \\
\hline & & & & & $\begin{array}{l}\text { pterocarp } \\
\text { um (DC.) }\end{array}$ & ceae & $\begin{array}{l}\text { popper } \\
\text { pod }\end{array}$ & leaf & $\begin{array}{l}\text { Brown, } \\
\text { black }\end{array}$ \\
\hline
\end{tabular}




\begin{tabular}{|l|l|l|l|l|}
\hline K.Heyne & & & & \\
\hline $\begin{array}{l}\text { Perilla } \\
\text { ocimoidea } \\
\text { L. }\end{array}$ & Labiatae & $\begin{array}{l}\text { Kumboo } \\
\text { millet }\end{array}$ & Fruit & Black \\
\hline $\begin{array}{l}\text { Pistacia } \\
\text { intergerri } \\
\text { ma } \text { L. }\end{array}$ & $\begin{array}{l}\text { Anacardiac } \\
\text { eae }\end{array}$ & $\begin{array}{l}\text { East } \\
\text { Indian } \\
\text { mastecha } \\
\text { e }\end{array}$ & $\begin{array}{l}\text { Flower, } \\
\text { leaf }\end{array}$ & Yellow \\
\hline $\begin{array}{l}\text { Toddalia } \\
\text { asiatica } \\
\text { (L.) Lam. }\end{array}$ & Rutaceae & $\begin{array}{l}\text { Wild } \\
\text { orange }\end{array}$ & Root & Yellow \\
\hline
\end{tabular}

\begin{tabular}{|c|c|c|c|c|}
\hline & & & $\begin{array}{l}\text { d bixin } \\
\text { comprise } \\
\text { s } 70-80 \% \\
\text { in each } \\
\text { seed. }\end{array}$ & $\begin{array}{l}\text { tropical } \\
\text { America, it } \\
\text { has become } \\
\text { naturalised in } \\
\text { the hotter } \\
\text { parts of } \\
\text { India. }\end{array}$ \\
\hline $\begin{array}{l}\text { Butea } \\
\text { monospe } \\
\text { rma } \\
\text { (Lam) } \\
\text { Taubert. }\end{array}$ & \begin{tabular}{l}
\multicolumn{2}{c}{ Yello } \\
$\mathrm{w}$ or \\
orange
\end{tabular} & Butrin & - & $\begin{array}{l}\text { Commonly } \\
\text { found } \\
\text { throughout } \\
\text { India, except } \\
\text { in the arid } \\
\text { region. It } \\
\text { grows on } \\
\text { black cotton } \\
\text { soil, even on } \\
\text { saline, } \\
\text { alkaline and } \\
\text { swampy } \\
\text { badly drained } \\
\text { soils and in } \\
\text { barren lands. }\end{array}$ \\
\hline $\begin{array}{l}\text { Cartham } \\
\text { us } \\
\text { tinctorio } \\
\text { us L. }\end{array}$ & $\begin{array}{l}\text { Yello } \\
\text { w, red }\end{array}$ & $\begin{array}{l}\text { Carthami } \\
\mathrm{n}\end{array}$ & $\begin{array}{l}\text { The chief } \\
\text { constitue } \\
\text { nt } \\
\text { carthamin } \\
\text { ranges } \\
\text { from } 3 \text { to } \\
6 \% \text { of the } \\
\text { flower. }\end{array}$ & $\begin{array}{l}\text { Cultivated } \\
\text { throughout } \\
\text { India. It } \\
\text { requires } \\
\text { fertile, } \\
\text { moisture-rete } \\
\text { ntive and } \\
\text { well-drained } \\
\text { soil. }\end{array}$ \\
\hline $\begin{array}{l}\text { Curcuma } \\
\text { longa L. }\end{array}$ & $\begin{array}{l}\text { Yello } \\
\mathrm{W}\end{array}$ & $\begin{array}{l}\text { Curcumi } \\
\mathrm{n}\end{array}$ & $\begin{array}{l}\text { Percentag } \\
\text { e of } \\
\text { curcumin } \\
\text { varies } \\
\text { from } 5.4 \\
\text { to } 8.7 .\end{array}$ & $\begin{array}{l}\text { Turmeric } \\
\text { grown } \\
\text { generally as } \\
\text { an annual } \\
\text { crop. It is } \\
\text { cultivable } \\
\text { from sea } \\
\text { level up to } \\
1200 \text { M. it } \\
\text { thrives in } \\
\text { will-drained, } \\
\text { fertile, sandy } \\
\text { and clayey, } \\
\text { black red } \\
\text { soil. }\end{array}$ \\
\hline $\begin{array}{l}\text { Indigofer } \\
a \\
\text { tinctoria } \\
\text { L. }\end{array}$ & Blue & $\begin{array}{l}\text { Indigotin, } \\
\text { Indican }\end{array}$ & $\begin{array}{l}\text { Indigotin } \\
\text { content } \\
\text { varies } \\
\text { according } \\
\text { to season } \\
\text { and age } \\
\text { of the } \\
\text { plant. } \\
\text { Best } \\
\text { grade } \\
\text { contains } \\
70-90 \% \\
\text { in dried } \\
\text { leaves. }\end{array}$ & $\begin{array}{l}\text { Distributed } \\
\text { commonly in } \\
\text { the tropical } \\
\text { region. }\end{array}$ \\
\hline
\end{tabular}

Here we have some plants which contain dye pigments with its amount of content, habitat and distribution.

Table 3. Pigments of dye yielding plants (R.Siva, 2007)

\begin{tabular}{|c|c|c|c|c|}
\hline Plant & $\begin{array}{l}\text { Colou } \\
\mathbf{r} \\
\text { obtain } \\
\text { ed }\end{array}$ & Pigment & $\begin{array}{l}\text { Dye } \\
\text { content }\end{array}$ & $\begin{array}{l}\text { Habitat and } \\
\text { distribution }\end{array}$ \\
\hline $\begin{array}{l}\text { Acacia } \\
\text { catechu } \\
\text { (L.f.) } \\
\text { Willd. }\end{array}$ & $\begin{array}{l}\text { Brown } \\
\text {, black }\end{array}$ & $\begin{array}{l}\text { Catechin, } \\
\text { catechuta } \\
\text { nic acid }\end{array}$ & $\begin{array}{l}\text { The chief } \\
\text { constitue } \\
\text { nts of the } \\
\text { heartwoo } \\
\text { d vary } \\
\text { from } 4 \text { to } \\
7 \% \text { and } \\
\text { are } \\
\text { distribute } \\
\text { d } \\
\text { througho } \\
\text { ut the } \\
\text { heartwoo } \\
\text { d from } \\
\text { the root to } \\
\text { the } \\
\text { branches. }\end{array}$ & $\begin{array}{l}\text { Occurs } \\
\text { throughout } \\
\text { India in dry } \\
\text { types of } \\
\text { mixed forest } \\
\text { on a variety } \\
\text { of geological } \\
\text { formations } \\
\text { and soils. }\end{array}$ \\
\hline $\begin{array}{l}\text { Adhatod } \\
a \quad \text { vasica } \\
\text { Nees. }\end{array}$ & $\begin{array}{l}\text { Yello } \\
\mathrm{W}\end{array}$ & $\begin{array}{l}\text { Adhatodi } \\
\text { c acid, } \\
\text { carotein, } \\
\text { lutolin, } \\
\text { quercetin }\end{array}$ & - & $\begin{array}{l}\text { Distributed } \\
\text { throughout } \\
\text { India, up to } \\
\text { an attitude of } \\
1300 \text { m; } \\
\text { grows on } \\
\text { waste land } \\
\text { and in a } \\
\text { variety of } \\
\text { habitats and } \\
\text { soil. It is } \\
\text { sometimes } \\
\text { cultivated as } \\
\text { hedge. }\end{array}$ \\
\hline $\begin{array}{l}\text { Bixa } \\
\text { orellena } \\
\text { L. }\end{array}$ & $\begin{array}{l}\text { Orang } \\
\text { e, red }\end{array}$ & $\begin{array}{l}\text { Bixin, } \\
\text { norbixin }\end{array}$ & $\begin{array}{l}\text { The dye } \\
\text { content is } \\
5-6 \% \text { by } \\
\text { weight of } \\
\text { seed. A } \\
\text { carotenoi }\end{array}$ & $\begin{array}{l}\text { the small tree } \\
\text { is found to } \\
\text { thrive at } \\
\text { elevations of } \\
600-900 \text { M; } \\
\text { native to }\end{array}$ \\
\hline
\end{tabular}




\section{Available online at www.ijrat.org}

\begin{tabular}{|c|c|c|c|c|c|c|c|c|c|}
\hline $\begin{array}{l}\text { lawsonia } \\
\text { inermis } \\
\mathrm{L} .\end{array}$ & $\begin{array}{l}\text { Orang } \\
\mathrm{e}^{\text {Pan }}\end{array}$ & Lawsone & $\begin{array}{l}\text { The } \\
\text { principle } \\
\text { colouring } \\
\text { matter, } \\
\text { lawsone } \\
\text { is present } \\
\text { in dried } \\
\text { leaves at } \\
\text { a } \\
\text { concentra } \\
\text { tion of } \\
1.0-1.4 \% .\end{array}$ & $\begin{array}{l}\text { It is mainly } \\
\text { cultivated in } \\
\text { Tamil Nadu, } \\
\text { Madhya } \\
\text { Prades and } \\
\text { Rajasthan. It } \\
\text { can grow on } \\
\text { any type of } \\
\text { soil from } \\
\text { light loam to } \\
\text { clay loam but } \\
\text { grows best } \\
\text { on heavy } \\
\text { soil. }\end{array}$ & $\begin{array}{l}\text { Punica } \\
\text { granatu } \\
m \mathrm{~L} .\end{array}$ & $\begin{array}{l}\text { Yello } \\
\mathrm{w}\end{array}$ & $\begin{array}{l}\text { Petargoni } \\
\text { don 3,5, } \\
\text { diglucosi } \\
\text { de }\end{array}$ & - & $\begin{array}{l}\text { Mostly found } \\
\text { cultivated in } \\
\text { many parts of } \\
\text { India. The } \\
\text { tree is also } \\
\text { common and } \\
\text { gregarious in } \\
\text { the gravel } \\
\text { and boulder } \\
\text { deposits of } \\
\text { dry ravines } \\
\text { and similar } \\
\text { places in the } \\
\text { outer }\end{array}$ \\
\hline \multirow{3}{*}{$\begin{array}{l}\text { Mallotus } \\
\text { philippen } \\
\text { is Muell. }\end{array}$} & \multirow[t]{3}{*}{ Red } & \multirow[t]{3}{*}{ Rottlerin } & \multirow{3}{*}{$\begin{array}{l}\text { The yield } \\
\text { of powder } \\
\text { rottlerin } \\
\text { is } \\
1.4-3.7 \% \\
\text { of the } \\
\text { weight of } \\
\text { the fresh } \\
\text { fruits. }\end{array}$} & \multirow{3}{*}{$\begin{array}{l}\text { Found } \\
\text { throughout } \\
\text { India; } \\
\text { occasionally } \\
\text { ascending to } \\
1500 \mathrm{~m} \text { in the } \\
\text { outer } \\
\text { Himalayas. } \\
\text { Commonly } \\
\text { found in Sal } \\
\text { and certain } \\
\text { shrub and } \\
\text { mixed } \\
\text { forests. }\end{array}$} & & & & & $\begin{array}{l}\text { Himalayas } \\
\text { up to } 1800 \mathrm{~m} \text {. }\end{array}$ \\
\hline & & & & & $\begin{array}{l}\text { Rubia } \\
\text { cordifoli } \\
a \mathrm{~L} .\end{array}$ & Red & Purpurin & $\begin{array}{l}\text { Purpurin } \\
\text { per cent } \\
\text { vary from } \\
2.0 \text { to } 4.0\end{array}$ & $\begin{array}{l}\text { A hardy } \\
\text { climber } \\
\text { common } \\
\text { thoughout } \\
\text { India, } \\
\text { ascending to } \\
\text { an altitude of } \\
3750 \mathrm{~m} \text {. }\end{array}$ \\
\hline & & & & & \multirow[t]{2}{*}{$\begin{array}{l}\text { Semecar } \\
\text { pus } \\
\text { anacardi } \\
\text { um L.f. }\end{array}$} & Black & $\begin{array}{l}\text { Bhilawan } \\
\text { ol }\end{array}$ & $\begin{array}{l}\text { Bhilawan } \\
\text { ol ranging } \\
\text { from } 28 \\
\text { to } 36 \% \text { of }\end{array}$ & $\begin{array}{l}\text { The tree is } \\
\text { common in } \\
\text { forests often } \\
\text { found }\end{array}$ \\
\hline \multirow[t]{2}{*}{$\begin{array}{l}\text { Morinda } \\
\text { citrifolia } \\
\text { L. }\end{array}$} & \multirow[t]{2}{*}{$\begin{array}{l}\text { Yello } \\
\text { w, red }\end{array}$} & \multirow[t]{2}{*}{$\begin{array}{l}\text { Morindo } \\
\text { ne }\end{array}$} & \multirow{2}{*}{$\begin{array}{l}\text { Roots are } \\
\text { guh out } \\
\text { when the } \\
\text { plants are } \\
3-4 \text { years } \\
\text { old, dried } \\
\text { and } \\
\text { sorted for } \\
\text { use by the } \\
\text { dyeing } \\
\text { trade. }\end{array}$} & \multirow[t]{2}{*}{$\begin{array}{l}\text { A small tree } \\
\text { distributed } \\
\text { throughtout } \\
\text { the tropics. }\end{array}$} & & & & $\begin{array}{l}\text { dry } \\
\text { weight of } \\
\text { seed. }\end{array}$ & $\begin{array}{l}\text { occuring } \\
\text { with Sal, } \\
\text { throughout } \\
\text { the hotter } \\
\text { parts if India. }\end{array}$ \\
\hline & & & & & $\begin{array}{l}\text { Toddalia } \\
\text { asiatica } \\
\text { (L.) Lam. }\end{array}$ & $\begin{array}{l}\text { Yello } \\
\mathrm{w}\end{array}$ & $\begin{array}{l}\text { Toddalin } \\
\mathrm{e}\end{array}$ & - & $\begin{array}{l}\text { In South } \\
\text { India, the } \\
\text { plant is } \\
\text { common in } \\
\text { the Nilgris } \\
\text { and Palani }\end{array}$ \\
\hline \multirow[t]{2}{*}{$\begin{array}{l}\text { Oldenlan } \\
\text { dia } \\
\text { umbellat } \\
\text { a L. }\end{array}$} & \multirow[t]{2}{*}{ Red } & \multirow[t]{2}{*}{$\begin{array}{l}\text { Alizarin, } \\
\text { Rubichol } \\
\text { ric acid }\end{array}$} & \multirow[t]{2}{*}{ 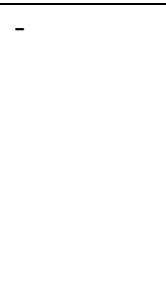 } & \multirow{2}{*}{$\begin{array}{l}\text { Prostrate } \\
\text { herb } \\
\text { distributed in } \\
\text { the tropical } \\
\text { and } \\
\text { subtropical } \\
\text { region. }\end{array}$} & & & & & $\begin{array}{l}\text { hills, and also } \\
\text { in } \\
\text { scrubby } \\
\text { jungles of } \\
\text { Orissa. } \\
\end{array}$ \\
\hline & & & & & \multirow{2}{*}{$\begin{array}{l}\text { Wrightia } \\
\text { tinctoria } \\
\mathrm{R} \text {. Br. }\end{array}$} & \multirow[t]{2}{*}{ Blue } & \multirow[t]{2}{*}{$\begin{array}{l}\beta \text {-amyrin } \\
\mathrm{e}\end{array}$} & \multirow{2}{*}{$\begin{array}{l}\text { Leaves } \\
\text { are the } \\
\text { source of } \\
\text { blue dye } \\
\text { called } \\
\text { Mysore } \\
\text { pala-Indi } \\
\text { go and } \\
\beta \text {-amyrin } \\
\text { e ranges } \\
\text { from } \\
3.3-5.0 \% \\
\text { of dried } \\
\text { leaves. }\end{array}$} & \multirow[b]{2}{*}{$\begin{array}{l}\text { Distributed } \\
\text { in Rajasthan, } \\
\text { Madhya } \\
\text { Pradesh and } \\
\text { peninsular } \\
\text { India, } \\
\text { ascending to } \\
\text { an altitude of } \\
1200 \mathrm{~m} \text { in the } \\
\text { hills. }\end{array}$} \\
\hline $\begin{array}{l}\text { Pterocar } \\
\text { pus } \\
\text { santalinu } \\
s \text { L. }\end{array}$ & Red & Santalin & $\begin{array}{l}\text { Red } \\
\text { sanders } \\
\text { contain } \\
16 \% \text { of a } \\
\text { colouring } \\
\text { matter, } \\
\text { santalin } \\
\text { (santalic } \\
\text { acid). }\end{array}$ & $\begin{array}{l}\text { Grows } \\
\text { typically of } \\
\text { dry, hilly, } \\
\text { often rocky } \\
\text { ground and is } \\
\text { occasionally } \\
\text { fround } \\
\text { growing on } \\
\text { precipitous } \\
\text { hillside. }\end{array}$ & & & & & \\
\hline
\end{tabular}


International Journal of Research in Advent Technology, Vol.8, No.3, March 2020

E-ISSN: 2321-9637

Available online at www.ijrat.org

Table 4. Plants and its Pigment's Chemical structure and Molecular formula

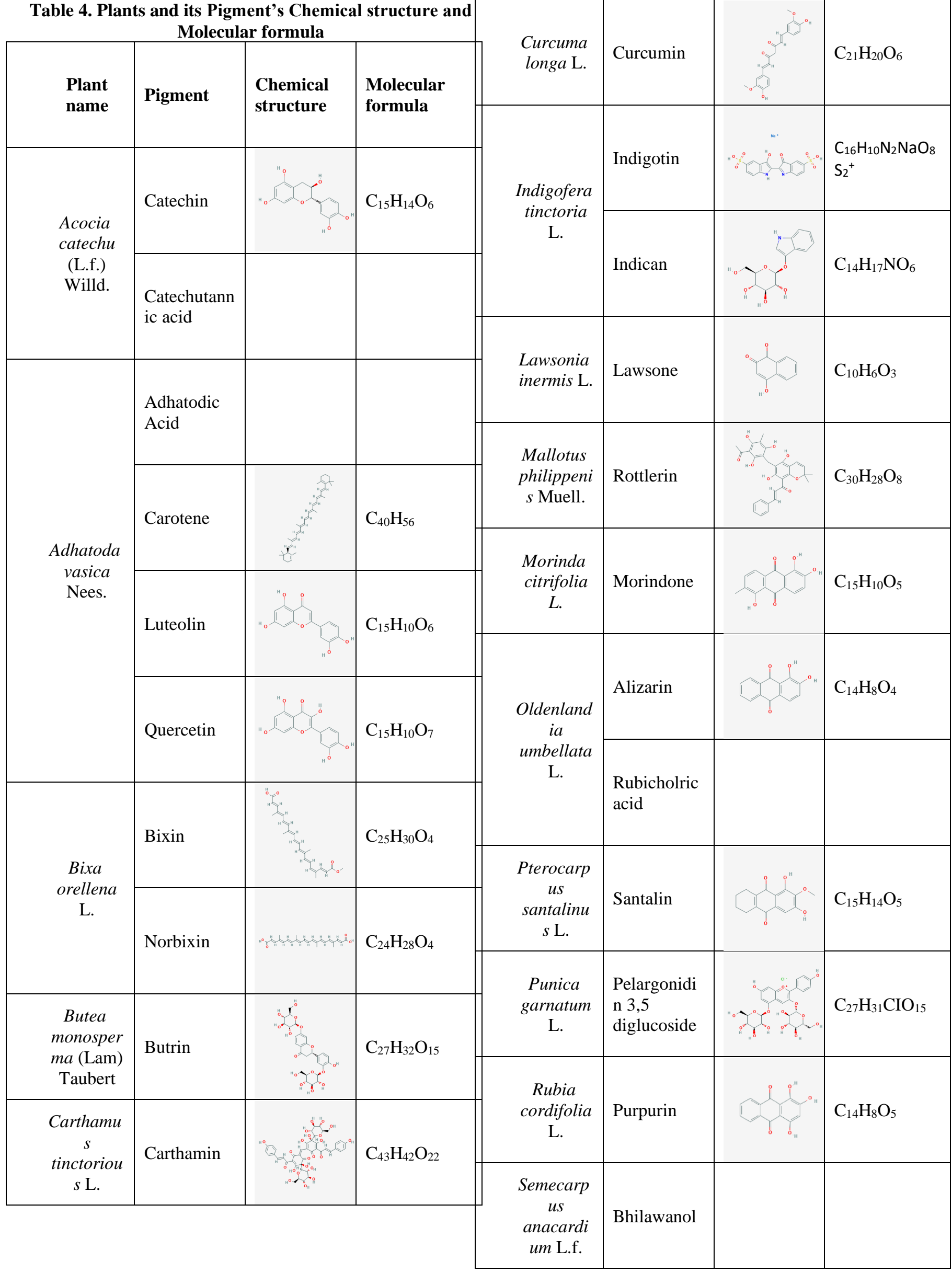




\begin{tabular}{|c|c|c|c|}
$\begin{array}{c}\text { Toddalia } \\
\text { asiatica } \\
\text { (L.) Lam. }\end{array}$ & Toddaline & & $\mathrm{C}_{21} \mathrm{H}_{18} \mathrm{NO}_{4}$ \\
\hline $\begin{array}{c}\text { Wrightia } \\
\text { tinctoria } \\
\text { R. Br. }\end{array}$ & $\beta$-amyrine & & \\
\hline
\end{tabular}

\section{PReParation OF DYES}

Two hundred years or more ago it was necessary to produce crops such as indigo and woad, weld and madder based on labour-intensive agronomy, and time-consuming downstream manufacturing processes to prepare the dye (Dogan Y, 2003, W Crokes, 1874, Krishnamurty T., 1993). All of that started dying out in the mid-19th century. Agriculture has since become a technologically advanced industry, yet our understanding of how to grow dye crops has become fossilized.

The dye is usually prepared by boiling water in the crushed powder, but sometimes it is left to soak in cold water. In general, the solution obtained then is used to dye coarse cotton fabrics. Alum is usually employed as a mordant. Butea monosperma (Lam) flowers at Taubert. Make an orange-coloured dye that is not quick and easy to wash away (R.Siva, 2007). The material is steeped in a hot or cold decoction of the flowers for colouring purposes. Whether by first preparing the cloth with alum and wood ash, or by adding these substances to the dye bath, a more permanent colour is created. The indigo dye is produced by steeping the plant in water and permitting fermentation. The solution is then oxidized with air in a separate vessel. Muell of Mallotus philippinensis. Provides an orange colour, used to dye silk and wool. Preparing the B Annatto Dye. Orellena L., when almost ripe, the fruits are harvested. The seeds and pulp are separated from the mature fruit, and water is macerated. These are either ground up in an' annatto paste' or dried and sold as annatto seeds afterwards. Sometimes when the seeds and pulp are macerated with water, the substance is stained through a sieve and the colouring content that settles out is collected and partly heat-evaporated and eventually dried in the light (B Glover and J H Pierce, 1993).

\section{Conclusion}

Natural dyes Vs Synthetic dyes \& Conclusion

Without particular conclusive evidence, several people in the textile industry have argued that natural colours are poor have fastness, that the colours produced are of poor quality, that the colours are expensive and difficult to use, and that there is not enough land to grow them (B. Glover, 1995, U Sewekow, 1988, U Sewekow, 1995, D J Hill, 1997). Therefore, they say that natural dyes are not a suitable commercial alternative to synthetic dyes, and therefore they have no future in the textile industry. But this is to miss the point regarding natural colouring, as no one suggests that natural colouring could completely replace synthetic colouring. Natural dyes have environmental and health issues that cannot be ignored. Nevertheless, synthetic colouring itself can pose significant industrial hazards, and its manufacturing is protected by strict COSHH (Control of Substances Hazardous to Health) regulations that are not needed for the production and processing of natural colorants.

\section{REFERENCES}

[1] R.Siva, (2007) Status of natural dyes and dye-yielding plants in India, Current science; Vol.92: 916-925.

[2] Gulrajani, M. L., (1992) Introduction to Natural Dyes, Indian Institute of Technology, New Delhi.

[3] Ozlenen Erdem Ismal, (2016) Patterns from Nature: Contact Printing, TEXTILE Association;81-91.

[4] M. L. Gulrajani, (2001) Present status of natural dyes, Indian Journal of Fibre \& Textile Research; Vol.26: 191-201.

[5] Siva, R., (2003) Assessment of genetic variation in some dye-yielding plants using isozyme data, $\mathrm{Ph} \mathrm{D}$ thesis, Bharathidasan University, Tiruchirapalli.

[6] Oktav Bulut M \& Akar E, (2012) Journal of Cleaner Production Vol.32.

[7] Vanker, P. S., (2000) Chemistry of natural dyes. Resonance, Vol.5: 73-80.

[8] W Blith, (1652) The English improver improved, London.

[9] Dogan Y, (2003) Economic Botany, Vol.57, 442

[10] P Miller, (1759) Gardeners' dictionary, $7^{\text {th }}$ Edn London.

[11] W Crokes, (1874) A practical handbook of dyeing and calico printing London.

[12] Krishnamurthy. T., (1993) Minor Forest Products of India, Oxford and IBH, New Delhi 340-364.

[13] B Glover and J H Pierce, (9193) J.S.D.C., Vol.109: 5.

[14] B Glover, (1995) Text. Chem. Colorist, Vol.27: 17.

[15] U Sewekow, (1988) Meiliand Textilber., Vol.4: 271.

[16] U Sewekow, (1995) Meiliand Textilber., Vol.5: 330.

[17] D J Hill, (1997) Is there a future for natural dyes? Rev. Prog. Coloration; Vol.27

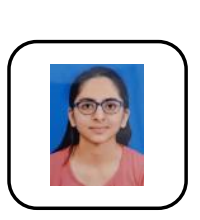

\section{AUTHORS PROFILE}

Maitri Kagathara, Department of Botany, Bioinformatics and Climate Change Impacts Management, Gujarat University, Ahmedabad, India.

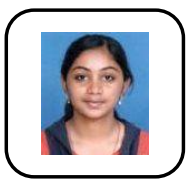

Dipika J. Dalal, Research Scholar, Department of Botany, Bioinformatics and Climate Change Impacts Management, Gujarat University, Ahmedabad, India.

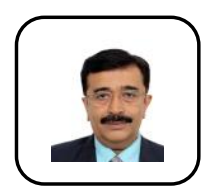

Dr. Hitesh A. Solanki, Department of Botany, Bioinformatics and Climate Change Impacts Management, Gujarat University, Ahmedabad, India. 